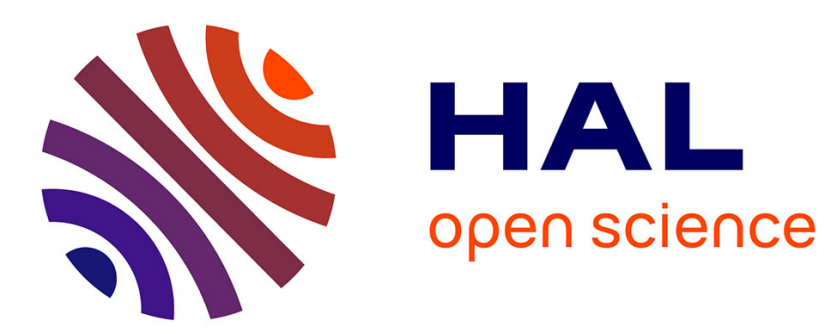

\title{
Discrete bandelets with geometric orthogonal filters
}

\author{
Gabriel Peyré, Stéphane Mallat
}

\section{To cite this version:}

Gabriel Peyré, Stéphane Mallat. Discrete bandelets with geometric orthogonal filters. IEEE International Conference on Image Processing, 2005. (ICIP 2005), Sep 2005, Genova, Italy. pp.65-68, 10.1109/ICIP.2005.1529688 . hal-00365624

\section{HAL Id: hal-00365624 \\ https://hal.science/hal-00365624}

Submitted on 3 Mar 2009

HAL is a multi-disciplinary open access archive for the deposit and dissemination of scientific research documents, whether they are published or not. The documents may come from teaching and research institutions in France or abroad, or from public or private research centers.
L'archive ouverte pluridisciplinaire $\mathbf{H A L}$, est destinée au dépôt et à la diffusion de documents scientifiques de niveau recherche, publiés ou non, émanant des établissements d'enseignement et de recherche français ou étrangers, des laboratoires publics ou privés. 


\title{
DISCRETE BANDELETS WITH GEOMETRIC ORTHOGONAL FILTERS
}

\author{
Gabriel Peyré, $\quad$ Stéphane Mallat \\ CMAP, École Polytechnique \\ 91128 Palaisau Cedex, France
}

\begin{abstract}
This paper describes the construction of second generation bandelet orthogonal bases. The decomposition on a bandelet basis is computed using a wavelet filter bank followed by adaptive geometric orthogonal filters, that require $O(N)$ operations. The resulting geometry is multiscale and calculated with a fast procedure that minimizes a Lagrangian cost at each scale. Image compression with the resulting bandelet transform code gives significantly better results than a wavelet transform code.
\end{abstract}

\section{INTRODUCTION}

\subsection{Geometry is Discrete and Multiscale}

Working with digital data means working in a discrete setting. A wavelet transform [1] can be applied on the discrete data to obtain a multiscale representation of the original data. In order to go one step further, today image processing algorithms try to exploit some geometrical regularity of the underlying function. But we must keep in mind that this geometry has to be defined in a discrete setting. In this paper we give a theoretic and algorithmic approach to compute such a geometric representation in the discrete wavelet domain. An application to image compression will be shown.

\subsection{Geometrically Regular Images}

Functions with geometric regularity are modeled as piecewise $\mathrm{C}^{\alpha}$-regular functions outside a set of edges which are themselves regular. However, natural images often do not have sharp discontinuities, so the model also includes some smoothing by an unknown kernel. The resulting functions can be written as a convolution $f=\widetilde{f} * h$ where $\widetilde{f}$ is a function with sharp features (regular outside a set of edges) and $h$ is the unknown smoothing kernel. We call this class of functions $\mathrm{C}^{\alpha}$-geometrically regular functions (see figure 1 , (a)).

Standard wavelet bases [1] are optimal to represent functions with pointwise singularities. However they fail to capture the geometric regularity along the singularities of a surfaces, because of their isotropic support. For $\mathrm{C}^{\alpha}$-geometrically regular functions, the distortion-rate of a wavelet image transform code with $R$ bits satisfies

$$
\left\|f-f_{R}\right\|^{2} \leqslant C R^{-1} \log (R) .
$$

To exploit the anisotropic regularity of a surface along edges, the basis must include elongated functions that are nearly parallel to the edges. Our goal is to build a new class of orthogonal bases for which the distortion rate has an optimal decay.

\subsection{Previous Works}

Several image representations have been proposed to capture geometric image regularity, and in particular curvelet frames [2] and first generation bandelet bases [3]. However, these constructions are not built directly in the discrete domain, and they do not provide a multiresolution representation of the geometry. In consequence, the implementation and the mathematical analysis is more involved and less efficient.

Contourlets [4] are also bases constructed with elongated basis functions using the combination of a multiscale and a directional filter bank. Our approach is different because it constructs a basis with a multiscale geometry that is adapted to the function that is represented. Asymptotically, the resulting bandelets are regular functions with a compact support, which is not the case of contourlets. Wedgeprints of [5] also provide a discrete compression scheme that is built on top of a wavelet decomposition. However, it does not define a new orthogonal basis but rather a scheme to perform a vector quantization of orthogonal wavelet coefficients, using a model of step edges.

\section{CONSTRUCTION OF A BANDELET BASIS}

The bandelet decomposition is computed with a geometric orthogonal transform that is applied on orthognal wavelet coefficients. It is thus computed with a wavelet filter bank followed by directional orthogonal filters. Each geometric direction leads to a different transform, and one can find the optimal set of filters using a best basis algorithm.

To describe the construction of the geometric orthogonal filters, we consider a wavelet transform at a fixed scale 
$2^{j}$ and we perform a zoom on the wavelet transform (by selecting a sub-square $S$ ), near a singularity (see figure 1). Observe that wavelet coefficients $\left\langle f, \psi_{j n}\right\rangle$ are samples of an underlying regularized function

$$
\left\langle f, \psi_{j n}\right\rangle=f * \psi_{j}\left(2^{j} n\right) \text { where } \psi_{j}(x)=\frac{1}{2^{j}} \psi\left(-2^{-j} x\right) .
$$

As a result, although the original function may be singular at edge locations, the wavelet coefficients are samples of a regularized function, obtained by convolving the original function $f$ with the "burring" kernel $\psi_{j}$ of width $2^{j}$.

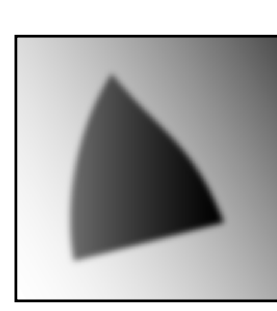

Original Image

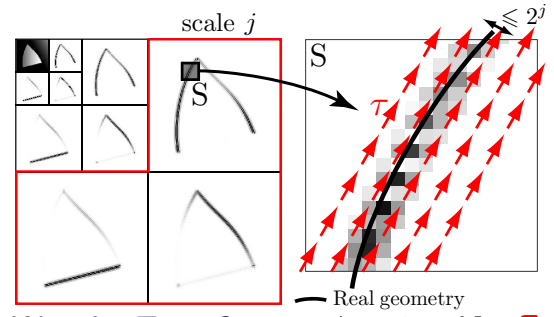

Wavelet Transform $\rightarrow$ Approximated flow $\tau$
Fig. 1. Zoom on the wavelet coefficients near a singularity.

In real applications the geometry must be estimated from the discrete image information. To make this problem more tractable, like in first generation bandelets, we

- search for a regularity flow (arrows) along which the function is "as regular as possible",

- search for a polynomial flow of degree $p-1$ parameterized either horizontally or vertically (on figure 1 one can see a linear flow).

Until the end of this section, we suppose that we defined a polynomial flow $\tau$ on some sub-square $S$ (we will later see how to find an optimal size for the squares and an optimal flow $\tau$ on each square).

If we impose that an integral curve of the geometric flow $\tau$ does not deviate more than $2^{j}$ (one pixel in wavelet space) from the real geometry, then one can prove an explicit bound of the derivatives of $f$ in the direction $\tau$ of the flow and in the vertical direction:

$$
\left|\frac{\partial^{a+b} f}{\partial \tau^{a} \partial y^{b}}\right| \leqslant C 2^{j} 2^{-a / \alpha j} 2^{-j b} \text {. }
$$

This control over the derivatives will drive the construction of a polynomial approximation for $f * \psi_{j}$ that is elongated and follows the flow in small "bands".

We want to approximate $f_{j}=f * \psi_{j}$ on the regular grid of spacing $2^{j}$, restricted to a small square $S$, knowing the approximate geometric flow $\tau$. In order to do so, we construct an orthogonal filter bank that will decompose $f_{j}$ on functions that follows the geometrical flow. The support of the filters is obtained using a dyadic grouping of the sampling points inside $S$ into bands $\left\{B_{m}^{k}\right\}_{m=1}^{2^{k}}$ for each filtering step $k$ (see figure 2). The exact filter responses are obtained using polynomials multiresolution restricted to the dyadic groups, and are in fact 2D extensions of Alpert multiwavelets. This orthogonal directional filter bank can be implemented using the fast linear-time algorithm of Alpert [6]. The underlined filters are calculated within the transform procedure using a Gram-Schmidt orthogonalization. The resulting filters have $p$ vanishing moments, as the original wavelet basis, but they are elongated along the singularity, whereas the original wavelets have a square support.
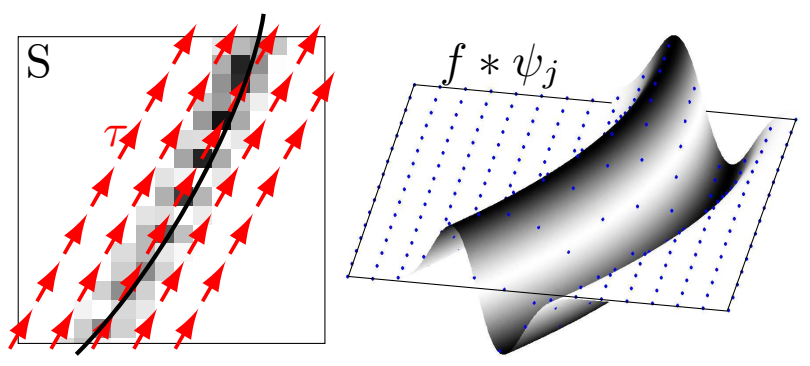

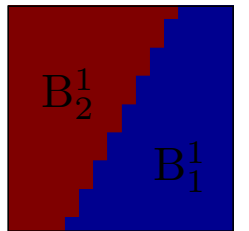

$k=1$

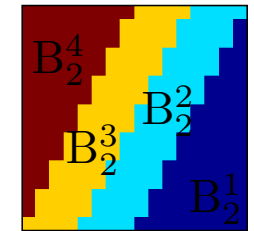

$k=2$

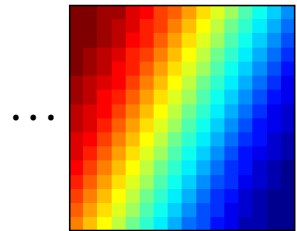

$k=5$
Fig. 2. $2 D$ and continous-3D plot of $f * \psi_{j}$ restricted to $S$ (top). Construction of a recursive binary partitions (bottom). Each $B_{m}^{k}$ is displayed in a different grey level.

To construct a bandelet basis on the whole wavelet domain, we use a quadtree segmentation of each wavelet scale in dyadic squares, as shown on figure 3 . Note that we define a different quadtree for each scale of the wavelet transform (on figure 3 only the quadtree of the finest scale is depicted), but we use the same quadtree for each of the three orientations of the wavelet transform at fixed scale. A complete representation in a bandelet basis $\mathscr{B}=\left\{b_{n}\right\}$ is thus composed of:

- An image segmentation at each scale $2^{j}$, defined by a quadtree.

- For each dyadic square of the segmentation,

- a polynomial flow,

- bandelet coefficients $\left\{\left\langle f, b_{n}\right\rangle\right\}_{n}$.

\section{BEST BASIS TRANSFORM CODE}

A bandelet transform code encodes the parameters that specify the geometry of the basis $\mathscr{B}=\left\{b_{n}\right\}_{n}$ that is adapted to the image, as well as the image coefficients in this bandelet basis. These bandelet coefficients are uniformly quantized with a step $T$ and a zero-bin twice larger corresponding to $[-T, T]$. The resulting coefficients are entropy coded with an arithmetic coder. At each scale $2^{j}$, the parameters of 

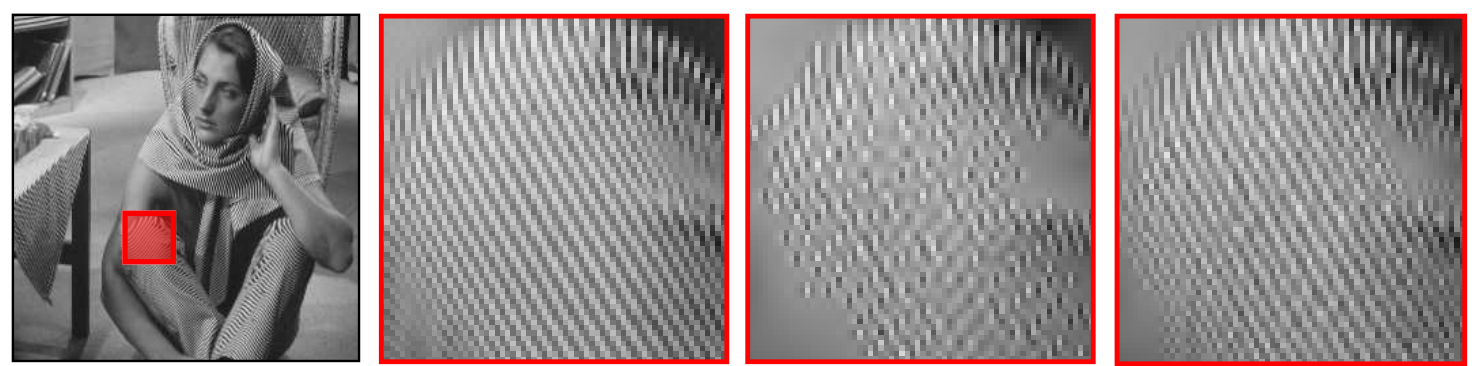

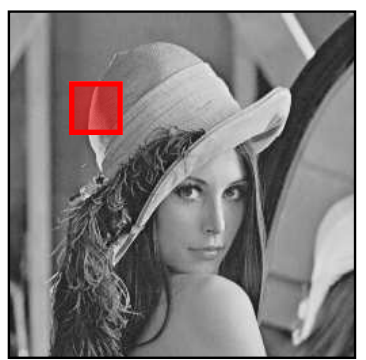

Original

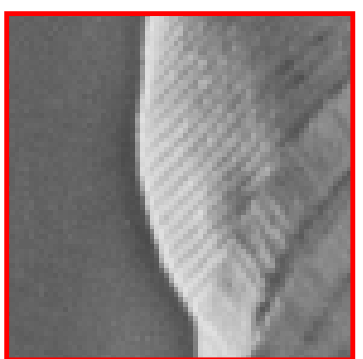

Original (zoom)
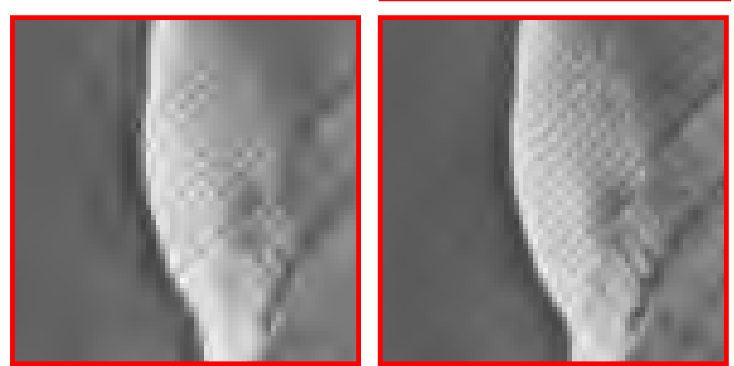

Wavelets at 0.2bpp Bandelets at 0.2bpp

Fig. 4. Comparison of Wavelets and Second Generation Bandelets at $0.2 \mathrm{bit} / \mathrm{pixel}$.

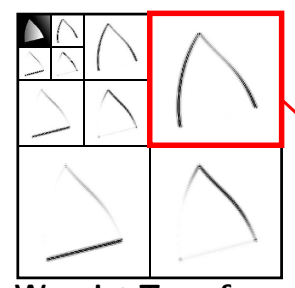

Wavelet Transform

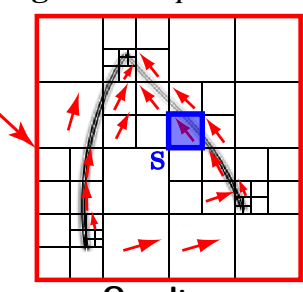

Quadtree

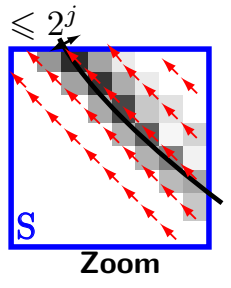

Zoom
Fig. 3. An example of quadtree segmentation of the wavelet space. Each segmentation define a bandelet basis. These geometric flows satisfy the error bound of $2^{j}$ (see zoom on right).

the geometry are also quantized to obtain a precision of $\left(2^{j}\right) / 4$. The total number of bits $R$ is decomposed into $R=\sum R_{j}=\sum\left(R_{j S}+R_{j G}+R_{j B}\right)$, where, for each scale $2^{j}$ :

- $R_{j S}$ is the number of bits to code the dyadic segmentation.

- $R_{j G}$ is the number of bits to code the quantized polynomial geometric flow in each square.

- $R_{j B}$ is the number of bits to code the quantized bandelet coefficients.

The image restored from its quantized bandelet coefficients is written $f_{R}$.

For a quantification step $T$, we would like to find the best basis $\mathscr{B}$ that minimizes the resulting distortion-rate. According to the approach in [3], we minimize a Lagrangian which can be shown to approximate the Lagrangian of the true distortion-rate:

$$
\mathscr{L}(f, R, \mathscr{B})=\left\|f-f_{R}\right\|^{2}+\frac{3}{4} T^{2} \sum_{j}\left(R_{S j}+R_{G j}+R_{B j}\right) .
$$

Thanks to the additivity of this Lagrangian and to the quadtree structure, the minimization of $\mathscr{L}$ can be performed using a fast CART-like bottom-up algorithm [3].
Even though we make an exhaustive search for the optimal geometry, for an image of $N$ pixels, the complexity of this best bandelet basis algorithm is $O\left(N^{3 / 2}\right)$ for a linear geometry (i.e. $p=2$ ). Some heuristics can also be used to reach a linear time complexity $O(N)$. The following theorem computes the asymptotic decay rate of the distortionrate obtained with this best bandelet basis transform code.
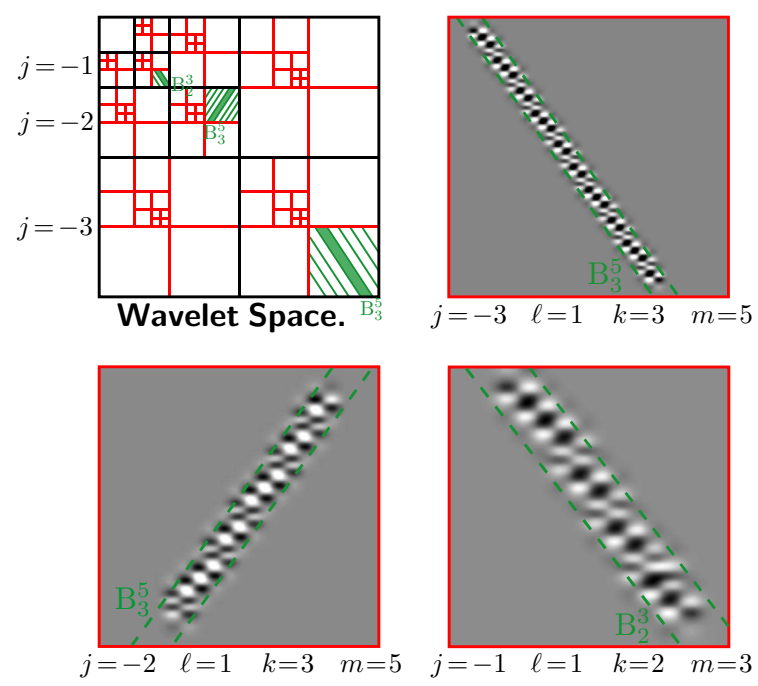

Fig. 5. Graphical display of continuous bandelet functions for various scales $j<0$, quad-tree levels $\ell$, partition depth $k$ and position $m$.

Theorem: Given $f \mathrm{a} \mathrm{C}^{\alpha}$-geometrically regular function, the transform coding $f_{R}$ with $R$ bits in the bandelet basis $\mathscr{B}$ minimizing $\mathscr{L}(f, R, \mathscr{B})$, with $R=R_{S}+R_{G}+R_{B}$, satisfies

$$
\left\|f-f_{R}\right\|^{2} \leqslant C \log ^{\alpha}(R) R^{-\alpha}
$$


with $C$ a constant that depends only on the function $f$.

We note the following important points:

- This exponent $\alpha$ is a priori unknown. The algorithm adapts itself to the best possible $\alpha$, and it is optimal.

- The reconstructed function is as regular as the original function (bandelet functions are regular, see figure 5).

- There is no blocking artifact due to the segmentation (in the spatial domain, bandelet functions do overlap with each other).

\section{NUMERICAL COMPRESSION RESULTS}

The bandelet transform is implemented with the 7/9 CDF biorthogonal wavelet basis. This best bandelet compression procedure was tested on various natural images. The PSNR improvement with respect to an equivalent wavelet transform coder is about $2 \mathrm{~dB}$ for Barbara and $0.6 \mathrm{~dB}$ for Lena (see figure 6). On figure 7 one can see, for the finest scale of the wavelet transform, the quadtree selected by the best basis algorithm, and a zoom on the orientation of the linear flow on each dyadic square. Figure 4 (upper row) shows a zoom on fine geometric structure of Barbara image, and one can see that Bandelets perform very well in these areas. The flow-based approach improves the encoding of these geometrically regular patterns.
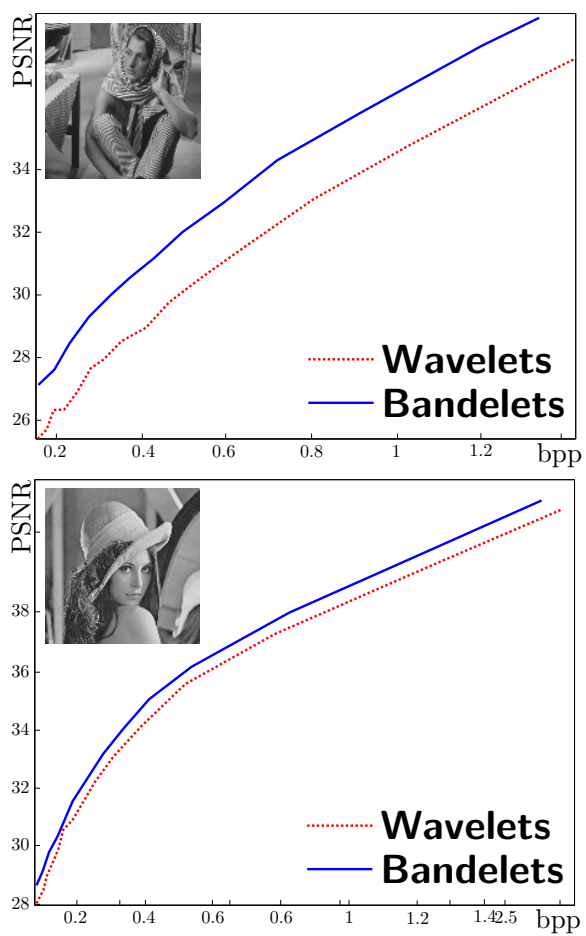

Fig. 6. Distortion curves for Lena (PSNR gain $\sim 0.6 \mathrm{~dB})$ and Barbara (PSNR gain $\sim 2 d B$ ).

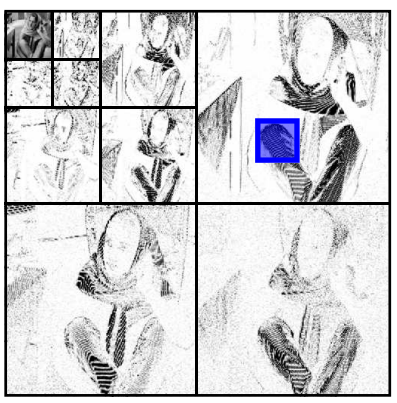

Wavelet transform

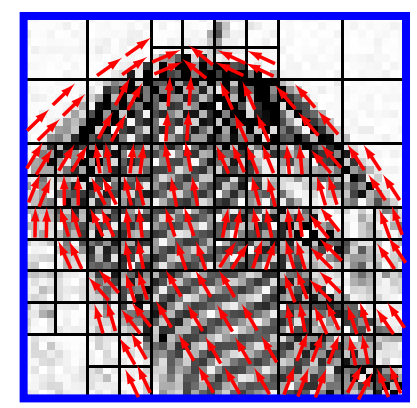

Segmentation and flow
Fig. 7. Wavelet coefficients and bandelets orientations at finest scale (quadtree and flow computed at 0.4 bit/pixel).

\section{CONCLUSION}

We introduced second generation bandelet bases that are entirely discrete and orthogonal and are calculated from a discrete wavelet basis. The construction uses a multi-scale description of the underlying geometry and the corresponding bandelets are regular. Image compression in a best bandelet basis provide a clear improvement over an equivalent transform coder in a wavelet basis.

\section{REFERENCES}

[1] S. Mallat, A Wavelet Tour of Signal Processing, Academic Press, San Diego, 1998.

[2] E. Candès and D. Donoho, Curvelets: A surprisingly effective nonadaptive representation of objects with edges, Vanderbilt Unervisity Press, 1999.

[3] E. Le Pennec and S. Mallat, "Sparse Geometrical Image Approximation with Bandelets," IEEE Transaction on Image Processing, 2004.

[4] M. N. Do and M. Vetterli, "The contourlet transform: an efficient directional multiresolution image representation,", To appear in IEEE Transactions Image on Processing, 2004.

[5] M. Wakin, J. Romberg, H. Choi, and R. Baraniuk, "Wavelet-domain Approximation and Compression of Piecewise Smooth Images," IEEE Transactions on Image Processing, November 2004, Submitted.

[6] B.K. Alpert, Wavelets and Other Bases for Fast Numerical Linear Algebra, C. K. Chui, editor, Academic Press, New York, 1992. 